\title{
TOEPLITZ OPERATORS ON WEIGHTED SPACES OF HOLOMORPHIC FUNCTIONS
}

\author{
ANAHIT HARUTYUNYAN* and WOLFGANG LUSKY
}

\begin{abstract}
We define a notion of Toeplitz operator on certain spaces of holomorphic functions on the unit disk and on the complex plane which are endowed with a weighted sup-norm. We establish boundedness and compactness conditions, give norm estimates and characterize the essential spectrum of these operators for many symbols.
\end{abstract}

\section{Introduction}

We deal with holomorphic functions $h: \Omega \rightarrow \mathrm{C}$, where $\Omega$ is the open unit disk $D=\{z \in C:|z|<1\}$ or $\Omega=C$, which are subject to certain growth conditions. To this end we consider an arbitrary function $v:\left[0, a\left[\rightarrow \mathbf{R}_{+}\right.\right.$ which is continuous and non-increasing where $a=1$ if $\Omega=\mathrm{D}$ and $a=\infty$ if $\Omega=$ C. If $a=1$ we assume that $\lim _{r \rightarrow 1} v(r)=0$ while for $a=\infty$ we assume that $\lim _{r \rightarrow \infty} r^{n} v(r)=0$ for any $n \geq 0$. $v$ is called a weight function. For fixed $r$ we put

$$
M_{\infty}(h, r)=\sup _{|z|=r}|h(z)| \quad \text { and } \quad\|h\|_{v}=\sup _{0 \leq r<a} M_{\infty}(h, r) v(r)
$$

and we define

$$
H v(\Omega)=\left\{h: \Omega \rightarrow C \text { holomorphic }:\|h\|_{v}<\infty\right\}
$$

$H v(\Omega)$ is a Banach space with the norm $\|\cdot\|_{v}$. We obtain $h \in H v(\Omega)$ if and only if $M_{\infty}(h, r)=O\left(\frac{1}{v(r)}\right)$ as $r \rightarrow a$. The conditions on $v$ ensure that $H v(\Omega)$ contains all polynomials.

The complete isomorphic classification of the spaces $H v(\Omega)$ is known ([1]). Indeed, $H v(\Omega)$ is either isomorphic to $l_{\infty}$ or to $H_{\infty}=\{h: \mathrm{D} \rightarrow$ C holomorphic : $h$ bounded\}. To decide whether $H v(\Omega)$ is isomorphic to $l_{\infty}$ one needs to consider the functions $\gamma_{n}(r)=r^{n} v(r)$ for any $n>0$. For each

\footnotetext{
* Supported by Deutsche Forschungsgemeinschaft 436 ARM 17/2/06.
}

Received December 31, 2006. 
$n>0$ pick a global maximum point $r_{n}$ of $\gamma_{n}$. We easily see that $\lim _{n \rightarrow \infty} r_{n}=a$. $v$ is said to satisfy condition (B) if

$$
\begin{gathered}
\forall b_{1}>1 \exists b_{2}>1 \exists c>0 \forall m, n>0: \\
\left(\frac{r_{m}}{r_{n}}\right)^{m} \frac{v\left(r_{m}\right)}{v\left(r_{n}\right)} \leq b_{1} \text { and } m, n,|m-n| \geq c \Rightarrow\left(\frac{r_{n}}{r_{m}}\right)^{n} \frac{v\left(r_{n}\right)}{v\left(r_{m}\right)} \leq b_{2}
\end{gathered}
$$

We have (see [2, Theorem 1.1])

$H v(\Omega)$ is isomorphic to $l_{\infty}$ if and only if $v$ satisfies $(\mathrm{B})$.

Examples of weights satisfying (B) include all normal weights on [0, 1[ (see [3]), in particular $v(r)=(1-r)^{\alpha}$ for any $\alpha>0$. Moreover $\exp (-1 /(1-$ $r)), \exp (-\exp (1 /(1-r))), \ldots$ satisfy $(\mathrm{B})$.

If $a=\infty$ then $\exp \left(-r^{\rho}\right)$ for any $\rho>0, \exp \left(-\log ^{\tau} r\right)$ for any $\tau \geq 2$, $\exp (-\exp (r)), \exp (-\exp (\exp (r))), \ldots$ satisfy $(\mathrm{B})$ (see [1] for details).

If $v$ satisfies (B) then $H v(\Omega)$ is complemented in any superspace. In this situation it is possible to give a meaningful definition of Toeplitz operator on $H v(\Omega)$. At first, we use induction to find indices $0<m_{1}<m_{2}<\cdots$ such that $r_{m_{1}}>0$ and

$$
3 \leq \min \left(\left(\frac{r_{m_{n}}}{r_{m_{n+1}}}\right)^{m_{n}} \frac{v\left(r_{m_{n}}\right)}{v\left(r_{m_{n+1}}\right)},\left(\frac{r_{m_{n+1}}}{r_{m_{n}}}\right)^{m_{n+1}} \frac{v\left(r_{m_{n+1}}\right)}{v\left(r_{m_{n}}\right)}\right) \leq 4
$$

(This is possible since, by assumption, $\lim _{M \rightarrow \infty} r_{M}^{n} v\left(r_{M}\right)=0$ for any $n>0$, see [1, Lemma 5.1].)

Let $g: \Omega \rightarrow C$ be a function such that $\left.g\right|_{r \partial \mathrm{D}}$ is continuous for each $r \in$ ]0, $a$ [. Then, for fixed $r$ let $\sum_{j \in Z} g_{j}(r) r^{|j|} e^{i j \varphi}$ be the Fourier series of $g$. For $0 \leq m<n$ put

$$
V_{n, m} g=\sum_{|j| \leq m} g_{j}(r) r^{|j|} e^{i j \varphi}+\sum_{m<|j| \leq n} \frac{[n]-|j|}{[n]-[m]} g_{j}(r) r^{|j|} e^{i j \varphi}
$$

where $[c]$ is the largest integer $\leq c$. In [2] the following theorem is shown (based on the results of [1])

THEOREM 1.1. If $v$ satisfies $(\mathrm{B})$ and $\left(m_{n}\right)$ are the preceding indices then there exist $d_{1}>0$ and $d_{2}>0$ such that, for any $h \in H v(\Omega)$, we have

$$
\begin{aligned}
& d_{1} \sup _{k} M_{\infty}\left(\left(V_{m_{k+1}, m_{k}}-V_{m_{k}, m_{k-1}}\right) h, r_{m_{k}}\right) v\left(r_{m_{k}}\right) \\
& \quad \leq\|h\|_{v} \leq d_{2} \sup _{k} M_{\infty}\left(\left(V_{m_{k+1}, m_{k}}-V_{m_{k}, m_{k-1}}\right) h, r_{m_{k}}\right) v\left(r_{m_{k}}\right)
\end{aligned}
$$


$\left(\right.$ Put $m_{0}=0$ and $V_{m_{0}, m_{-1}}=0$.)

This gives rise to the following definition. Let $g$ be as in the definition of $V_{n, m}$ and let $t_{k, j}$ be such that

$$
\left(V_{m_{k+1}, m_{k}}-V_{m_{k}, m_{k-1}}\right) g=\sum_{m_{k-1}<|j| \leq m_{k+1}} t_{k, j} g_{j}(r) r^{|j|} e^{i j \varphi} .
$$

Then define

$$
W_{k} g=\sum_{m_{k-1}<j \leq m_{k+1}} t_{k, j} g_{j}\left(r_{m_{k}}\right) r^{j} e^{i j \varphi}, \quad k \in \mathbf{Z}_{+}, \quad \text { and } \quad P g=\sum_{k} W_{k} g
$$

provided the last definition makes sense (i.e. the preceding Fourier series represents a holomorphic function on $\Omega$ ). One can show that $P g \in H v(\Omega)$ if $\sup _{0 \leq r<a} M_{\infty}(g, r) v(r)<\infty$ ([2]). It is easily seen that $P g=g$ if $g \in H v(\Omega)$.

Now we define Toeplitz operators.

Definition 1.2. Let $f: \Omega \rightarrow \mathrm{C}$ be such that, for all $r \in] 0, a\left[,\left.f\right|_{r \partial \mathrm{D}}\right.$ is continuous. For $h \in H v(\Omega)$ put

$$
T_{f}(h)=P(f h)
$$

(if this definition makes sense). Then $T_{f}$ is called Toeplitz operator with symbol $f$.

Later on (Corollary 2.6.) we show that for many symbols $f$ the definition of $T_{f}$ is independent of the numbers $m_{k}$ up to compact perturbations.

In section 2 we give boundedness and compactness conditions for $T_{f}$ and discuss the case of functions $f: \overline{\mathrm{D}} \rightarrow \mathrm{C}$ which are continuous on $\overline{\mathrm{D}} \backslash \rho \mathrm{D}$ for some $0<\rho<1$. In particular we show that, for suitable harmonic $g, T_{f}-T_{g}$ is compact. In section 4 we determine the essential spectrum for $T_{f}$ with respect to such functions $f$. Moreover, we show that, for harmonic $g: \mathrm{D} \rightarrow \mathrm{C},\left\|T_{g}\right\|$ and $M_{\infty}(g, 1)$ are equivalent.

\section{Continuity and compactness conditions for Toeplitz operators}

Again in this section let $f: \Omega \rightarrow C$ be such that

$$
\left.\left.f\right|_{r \partial r \mathrm{D}} \text { is continuous for all } r \in\right] 0, a[\text {. }
$$

Then, for each $r \in] 0, a\left[, f\left(r e^{i \varphi}\right)\right.$ has a Fourier series $\sum_{j} f_{j}(r) r^{|j|} e^{i j \varphi}$. For $0<p$ let the Cesaro mean $\sigma_{p}$ be defined by

$$
\sigma_{p} f=\sum_{|j| \leq p} \frac{[p]-|j|}{[p]} f_{j}(r) r^{|j|} e^{i j \varphi} .
$$


Note that

$$
M_{\infty}\left(\sigma_{p} f, r\right) \leq M_{\infty}(f, r) \quad \text { and } \quad \lim _{p \rightarrow \infty} M_{\infty}\left(f-\sigma_{p} f, r\right)=0
$$

for each $r$.

If $m_{k}$ are the preceding indices then fix $n_{k}$ such that

$$
0<\inf _{k}\left(\frac{m_{k+1}-m_{k}}{n_{k}}\right) \leq \sup _{k}\left(\frac{m_{k+1}-m_{k}}{n_{k}}\right)<\infty .
$$

We show

TheOREM 2.1. Let $f: \Omega \rightarrow C$ satisfy (2.1) and assume that

$$
\sup _{k} M_{\infty}\left(\sigma_{n_{k}}|f|, r_{m_{k}}\right)<\infty .
$$

Then $T_{f}$ is a bounded operator $H v(\Omega) \rightarrow H v(\Omega)$. Moreover there is a constant $c>0$ (independent of $f$ ) such that

$$
\left\|T_{f}\right\| \leq c \sup _{k} M_{\infty}\left(\sigma_{n_{k}}|f|, r_{m_{k}}\right) .
$$

We shall prove Theorem 2.1 in section 3. Using the preceding theorem we easily find examples even of unbounded $f: \Omega \rightarrow \mathrm{C}$ where $T_{f}: H v(\Omega) \rightarrow$ $H v(\Omega)$ is bounded.

ExAmple. Put

$$
f(z)= \begin{cases}\frac{1}{z^{n}}, & z \neq 0 \\ 0, & z=0\end{cases}
$$

for some integer $n>0$. Then, according to Theorem 2.1, $T_{f}$ is bounded and $\left\|T_{f}\right\| \leq c r_{m_{1}}^{-n}$.

In section 3 we also show

TheOREM 2.2. Let $f: \Omega \rightarrow$ C satisfy (2.1). If $\lim _{k \rightarrow \infty} M_{\infty}\left(\sigma_{n_{k}}|f|, r_{m_{k}}\right)=$ 0 then $T_{f}: H v(\Omega) \rightarrow H v(\Omega)$ is compact.

In the rest of this section we discuss some consequences of Theorem 2.2 for $\Omega=D$.

Proposition 2.3. Let $f: \overline{\mathrm{D}} \rightarrow \mathrm{C}$ satisfy (2.1) and assume that, for some $0<\rho<1$, $f$ is continuous on $\overline{\mathrm{D}} \backslash \rho \mathrm{D}$. Then

$$
\lim _{n \rightarrow \infty} \sup _{\rho \leq r \leq 1} M_{\infty}\left(f-\sigma_{n} f, r\right)=0
$$


In particular, $\lim _{n \rightarrow \infty}\left\|T_{f}-T_{\sigma_{n} f}\right\|=0$.

Proof. Using the Weierstraß theorem we see that $f$ can be uniformly approximated on $\backslash \rho \mathrm{D}$ by functions $g_{m}$ of the form

$$
g_{m}\left(r e^{i \varphi}\right)=\sum_{|k| \leq m} g_{m, k}(r) r^{|k|} e^{i k \varphi} .
$$

So fix $\epsilon>0$ and $g_{m}$ such that $M_{\infty}\left(f-g_{m}, r\right)<\epsilon$ for all $\rho \leq r \leq 1$. Hence

$$
\begin{aligned}
\sup _{\rho \leq r \leq 1} M_{\infty}\left(f-\sigma_{n} f, r\right) \leq & \sup _{\rho \leq r \leq 1} M_{\infty}\left(f-g_{m}, r\right)+\sup _{\rho \leq r \leq 1} M_{\infty}\left(\sigma_{n} g_{m}-\sigma_{n} f, r\right) \\
& \quad+\sup _{\rho \leq r \leq 1} M_{\infty}\left(g_{m}-\sigma_{n} g_{m}, r\right) \\
& <3 \epsilon
\end{aligned}
$$

for suitably large $n$. Then $\lim _{n \rightarrow \infty} \sup _{\rho \leq r \leq 1} M_{\infty}\left(f-\sigma_{n} f, r\right)=0$.

Let $k_{0}$ be such that $r_{m_{k}}>\rho$ for all $k \geq k_{0}$. Theorem 2.1. implies

$$
\begin{aligned}
\left\|T_{f}-T_{\sigma_{n} f}\right\| & =\left\|T_{f-\sigma_{n} f}\right\| \leq c \sup _{k} M_{\infty}\left(f-\sigma_{n} f, r_{m_{k}}\right) \\
& \leq c \max \left(\sup _{k \leq k_{0}} M_{\infty}\left(f-\sigma_{n} f, r_{m_{k}}\right), \sup _{\rho \leq r \leq 1} M_{\infty}\left(f-\sigma_{n} f, r\right)\right)
\end{aligned}
$$

In view of (2.1) this proves $\lim _{n \rightarrow \infty}\left\|T_{f}-T_{\sigma_{n} f}\right\|=0$.

Let $f$ satisfy the assumptions of Proposition 2.3. Then $\left.f\right|_{\partial \mathrm{D}}$ is continuous and has a harmonic extension $f_{h}$ on $\mathrm{D}$. So, if

$$
\gamma_{k}=\frac{1}{2 \pi} \int_{0}^{2 \pi} f\left(e^{i \varphi}\right) e^{-i k \varphi} d \varphi, \quad k \in \mathbf{Z}
$$

then $f_{h}\left(r e^{i \varphi}\right)=\sum_{k \in \mathrm{Z}} \gamma_{k} r^{|k|} e^{i k \varphi}, r<1$.

Corollary 2.4. Let $f: \overline{\mathrm{D}} \rightarrow \mathrm{C}$ satisfy (2.1) and assume that $f$ is continuous on $\overline{\mathrm{D}} \backslash \rho \mathrm{D}$ for some $0<\rho<1$. Then $T_{f}-T_{f_{h}}$ is compact.

Proof. In view of Proposition 2.3 it suffices to assume $f\left(r e^{i \varphi}\right)=$ $f_{j}(r) r^{|j|} e^{i j \varphi}$ for some $j \in \mathrm{Z}$. Then $\left|f-f_{h}\right|\left(r e^{i \varphi}\right)=\left.\left|f_{j}(r)-f_{j}(1)\right|\right|^{|j|}$. Using the numbers $n_{k}$ and $m_{k}$ of Theorems 2.1 and 2.2 we obtain

$$
\lim _{k \rightarrow \infty} M_{\infty}\left(\sigma_{n_{k}}\left|f-f_{h}\right|, r_{m_{k}}\right)=\lim _{k \rightarrow \infty}\left|f_{j}\left(r_{m_{k}}\right)-f_{j}(1)\right|=0 .
$$

Now Theorem 2.2 proves the corollary. 
Let $f\left(r e^{i \varphi}\right)=\sum_{|k| \leq n} f_{k}(r) r^{|k|} e^{i k \varphi}$ where all $f_{k}$ are continuous on $[\rho, 1]$ for some $0<\rho<1$. Put

$$
h_{f}(z)=\sum_{|k| \leq n} f_{k}(1) z^{k}
$$

Then $h_{f}$ is continuous on $\overline{\mathrm{D}} \backslash\{0\}$ and $\left(h_{f}\right)_{h}=f_{h}$. Corollary 2.4 implies

Lemma 2.5. Let $f: \overline{\mathrm{D}} \rightarrow \mathrm{C}$ satisfy (2.1) and assume that $f$ is continuous on $\overline{\mathrm{D}} \backslash \rho \mathrm{D}$ for some $0<\rho<1$. Then $T_{\sigma_{n} f}-T_{h_{\sigma_{n}}}$ is compact for all $n>0$.

The Toeplitz operator $T_{f}$ is defined via numbers $m_{k}$ satisfying (1.1). Let us go over to numbers $\tilde{m}_{k}$ which also satisfy (1.1) and consider the resulting Toeplitz operator $\tilde{T}_{f}$. Quite often we obtain that $T_{f}-\tilde{T}_{f}$ is compact.

Corollary 2.6. Let $f: \overline{\mathrm{D}} \rightarrow \mathrm{C}$ satisfy (2.1) and assume that $f$ is continuous on $\overline{\mathrm{D}} \backslash \rho \mathrm{D}$ for some $0<\rho<1$. Then $T_{f}$ is independent of the numbers $m_{k}$ up to compact perturbations.

Proof. In view of Proposition 2.3 and Lemma 2.5 it suffices to asume $f(z)=\alpha z^{n}$ for some $\alpha \in \mathrm{C}$ and $n \in \mathrm{Z}$. If $h \in H v(\mathrm{D})$ is such that $h(z)=$ $\sum_{k>|n|} \beta_{k} z^{k}$ then $f h$ is holomorphic. Hence if $T_{f}$ and $\tilde{T}_{f}$ are the Toeplitz operators with respect to the numbers $m_{k}$ and $\tilde{m}_{k}$ then $\left(T_{f}-\tilde{T}_{f}\right) h=0$. It follows that $T_{f}-\tilde{T}_{f}$ has finite rank and, therefore, is compact.

\section{Proofs of Theorems 2.1 and 2.2}

The proof follows from some lemmas. At first, let $f: \Omega \rightarrow C$ be such that $\left.f\right|_{r \partial \mathrm{D}} \in L_{1}(r \partial \mathrm{D})$ for all $r$ and the Fourier series of $f$ for fixed $r$ is $\sum_{j \in Z} f_{j}(r) r^{|j|} e^{i j \varphi}$. Let $R$ be the Riesz projection, i.e. $R f$ has the Fourier series $\sum_{j \geq 0} f_{j}(r) r^{|j|} e^{i j \varphi}$. Finally, for $k \in \mathrm{Z}$, define the translation operator $U_{k}$ by $U_{k} f=e^{i k \varphi} f$.

In [1, Lemma 3.3], it was shown that

$$
M_{\infty}\left(R\left(V_{p, n}-V_{n, m}\right) f, r\right) \leq \delta M_{\infty}(f, r)
$$

for all $r$ and $m, n, p \in \mathbf{Z}_{+}$with $0 \leq m \leq n \leq p$ where $\delta$ is a constant which depends only on

$$
\frac{p-m}{\min (p-n, n-m, m)}
$$

but not on $f$ or $r$. 
Lemma 3.1. Let $m, n, p \in \mathbf{Z}_{+}$such that $0 \leq m \leq n \leq p$ and fix $q \in \mathbf{Z}_{+}$. There is a universal constant $d>0$ depending only on

$$
\frac{p-m}{\min (p-n, n-m, m, q)}
$$

(but not on $f$ ) such that

$$
M_{\infty}\left(R\left(V_{p, n}-V_{n, m}\right) f, r\right) \leq d \sup _{k \in Z_{+}} M_{\infty}\left(U_{k} \sigma_{q} U_{-k} f, r\right) \quad \text { for all } r .
$$

Proof. For $k \geq q$ we have

$$
U_{k} \sigma_{q} U_{-k} f=\sum_{j=k-q}^{k} f_{j}(r) \frac{j+q-k}{q} r^{j} e^{i j \varphi}+\sum_{j=k+1}^{k+q} f_{j}(r) \frac{q+k-j}{q} e^{i j \varphi} .
$$

Hence we find $k_{1}, \ldots, k_{N}$ with

$$
R\left(V_{p, n}-V_{n, m}\right) \sum_{l=1}^{N} U_{k_{l}} \sigma_{q} U_{-k_{l}} f=R\left(V_{p, n}-V_{n, m}\right) f
$$

where $N$ depends only on $(p-m) / q$. This implies, with the previous constant $\delta$,

$$
M_{\infty}\left(R\left(V_{p, n}-V_{n, m}\right) f, r\right) \leq N \delta \sup _{k \in Z_{+}} M_{\infty}\left(U_{k} \sigma_{p-n} U_{-k} f, r\right)
$$

for any $r$ which proves the lemma.

Now we return to the definition of $W_{k}$ (preceding 1.2).

Lemma 3.2. There is a constant $c>0$ such that, for any $f: \Omega \rightarrow C$, $h \in H v(\Omega)$ and any $l \in \mathbf{Z}_{+}$, we have

$$
\left\|\sum_{k \geq l} W_{k}(f h)\right\|_{v} \leq c \sup _{k \geq l} M_{\infty}\left(W_{k}(f h), r_{m_{k}}\right) v\left(r_{m_{k}}\right) .
$$

Proof. By definition we have

$$
\left(V_{m_{j+1}, m_{j}}-V_{m_{j}, m_{j-1}}\right) W_{k}=0 \quad \text { if } \quad|k-j|>1 .
$$

Since $v$ satisfies (B) then either

$$
0<\inf _{k} \frac{m_{k+1}-m_{k}}{m_{k}-m_{k-1}} \leq \sup _{k} \frac{m_{k+1}-m_{k}}{m_{k}-m_{k-1}}<\infty
$$


or $\sup _{k}\left(m_{k+1}-m_{k-1}\right)<\infty$ ([1, Proposition 4.1]). According to [1, Lemma 3.3], we obtain that

$$
M_{\infty}\left(\left(V_{m_{j+1}, m_{j}}-V_{m_{j}, m_{j-1}}\right) g, r\right) \leq d M_{\infty}(g, r)
$$

for any $g$ and any $j$ where $d$ is a universal constant. Theorem 1.1 yields

$$
\begin{aligned}
& \left\|\sum_{k \geq l} W_{k}(f h)\right\|_{v} \\
& \leq d_{2} \sup _{j} M_{\infty}\left(\left(V_{m_{j+1}, m_{j}}-V_{m_{j}, m_{j-1}}\right)\left(\sum_{k \geq l} W_{k}\right)(f h), r_{m_{j}}\right) v\left(r_{m_{j}}\right) \\
& =d_{2} \sup _{j} M_{\infty}\left(\left(V_{m_{j+1}, m_{j}}-V_{m_{j}, m_{j-1}}\right)\left(W_{j-1}+W_{j}+W_{j+1}\right)(f h), r_{m_{j}}\right) v\left(r_{m_{j}}\right) \\
& \leq 3 d_{2} d \sup _{j \geq l} \max \left(M_{\infty}\left(W_{j}(f h), r_{m_{j-1}}\right) v\left(r_{m_{j-1}}\right),\right. \\
& \left.\quad M_{\infty}\left(W_{j}(f h), r_{m_{j}}\right) v\left(r_{m_{j}}\right), M_{\infty}\left(W_{j}(f h), r_{m_{j+1}}\right) v\left(r_{m_{j+1}}\right)\right)
\end{aligned}
$$

$W_{j}(f h)$ is a polynomial of the form $\sum_{k=m_{j-1}}^{m_{j+1}} \alpha_{k} z^{k}$. According to [1, Lemma 3.1], we infer

$$
\begin{aligned}
& M_{\infty}\left(W_{j}(f h), r_{m_{j-1}}\right) v\left(r_{m_{j-1}}\right) \\
& \leq 2\left(\frac{r_{m_{j-1}}}{r_{m_{j}}}\right)^{m_{j-1}} \frac{v\left(r_{m_{j-1}}\right)}{v\left(r_{m_{j}}\right)} M_{\infty}\left(W_{j}(f h), r_{m_{j}}\right) v\left(r_{m_{j}}\right)
\end{aligned}
$$

and

$$
\begin{aligned}
& M_{\infty}\left(W_{j}(f h), r_{m_{j+1}}\right) v\left(r_{m_{j+1}}\right) \\
& \leq 2\left(\frac{r_{m_{j+1}}}{r_{m_{j}}}\right)^{m_{j+1}} \frac{v\left(r_{m_{j+1}}\right)}{v\left(r_{m_{j}}\right)} M_{\infty}\left(W_{j}(f h), r_{m_{j}}\right) v\left(r_{m_{j}}\right)
\end{aligned}
$$

This yields the lemma.

To finish the proof of Theorem 2.1 consider, for $l>0$, the Fejer kernel

$$
F_{l}(\varphi)=\sum_{|j| \leq l} \frac{[l]-|j|}{[l]} e^{i j \varphi} .
$$

It is well-known that $F_{l}(\varphi) \geq 0$ for all $\varphi$. We have

$$
\left(\sigma_{l} f\right)\left(r e^{i \varphi}\right)=\frac{1}{2 \pi} \int_{0}^{2 \pi} F_{l}(\varphi-\psi) f\left(r e^{i \psi}\right) d \psi .
$$


Hence, if $h \in H v(\Omega)$ we obtain

$$
M_{\infty}\left(U_{k} \sigma_{l} U_{-k}(f h), r\right) \leq M_{\infty}\left(\sigma_{l}|f|, r\right) M_{\infty}(h, r)
$$

for any $k \in \mathbf{Z}$ and any $r>0$.

Conclusion of the proof of Theorem 2.1

We take into account that, for arbitrary $0<n<p$, we have $V_{p, n}=V_{[p],[n]}$. Assume $\sup _{k} M_{\infty}\left(\sigma_{n_{k}}|f|, r_{m_{k}}\right)<\infty$. With Lemma 3.1 we obtain constants $d_{k}$ such that, for any $h \in H v(\Omega)$,

$$
\begin{aligned}
M_{\infty}\left(W_{k}(f h), r_{m_{k}}\right) & \leq d_{k} \sup _{l \in Z_{+}} M_{\infty}\left(U_{l} \sigma_{n_{k}} U_{-l}(f h), r_{m_{k}}\right) \\
& \leq d_{k} M_{\infty}\left(\sigma_{n_{k}}|f|, r_{m_{k}}\right) M_{\infty}\left(h, r_{m_{k}}\right)
\end{aligned}
$$

Since $v$ satisfies (B) then either

$$
0<\inf _{k} \frac{m_{k+1}-m_{k}}{m_{k}-m_{k-1}} \leq \sup _{k} \frac{m_{k+1}-m_{k}}{m_{k}-m_{k-1}}<\infty
$$

or $\sup _{k}\left(m_{k+1}-m_{k-1}\right)<\infty([1$, Proposition 4.1]).

Hence, in view of Lemma 3.1, the $d_{k}$ are uniformly bounded. According to Lemma 3.2 with $l=1$ we obtain

$$
\left\|T_{f} h\right\|_{v} \leq\left(\sup _{k} d_{k}\right)\left(\sup _{k} M_{\infty}\left(\sigma_{n_{k}}|f|, r_{m_{k}}\right)\right)\|h\|_{v}
$$

Since $T_{f} h$ is a holomorphic function on $\Omega$ we conclude $T_{f} h \in H v(\Omega)$.

Conclusion of the proof of Theorem 2.2

Assume that $\lim _{k \rightarrow \infty} M_{\infty}\left(\sigma_{n_{k}}|f|, r_{m_{k}}\right)=0$. By the same argument as in the preceding proof, in view of Lemma 3.2, we see that, for any $\epsilon>0$, there is $l$ such that $\left\|\sum_{k>l} W_{k}(f h)\right\|_{v} \leq \epsilon\|h\|_{v}$ for any $h \in H v(\Omega)$. Hence $\| T_{f}(h)-$ $\sum_{k=1}^{l} W_{k}(f h)\left\|_{v} \leq \epsilon\right\| h \|_{v}$ for all $h \in H v(\Omega)$. This means that $T_{f}$ is the limit of a sequence of finite rank operators. Hence $T_{f}$ is compact.

\section{Some consequences}

Here we give more applications of the results of section 2 for the case $\Omega=D$. At first we show

LeMma 4.1. Let $h_{n}(z)=z^{n}, n \in \mathbf{Z}_{+}$. Then $h_{n} /\left\|h_{n}\right\|$ tends to 0 weakly (in $H v(\Omega))$. 
Proof. Consider

$$
(H v)_{0}(\Omega)=\left\{h: \Omega \rightarrow C: h \text { holomorphic, } \limsup _{r \rightarrow a|z|=r}|h(z)| v(r)=0\right\}
$$

and take the indices $m_{k}$ of Theorem 1.1 Put

$$
H_{k}=\operatorname{span}\left\{z^{j}: m_{k-1} \leq j \leq m_{k+1}\right\} \subset(H v)_{0}(\Omega) .
$$

Then Theorem 1.1 yields that $(H v)_{0}(\Omega) \subset\left(\sum_{k=1}^{\infty} \oplus H_{k}\right)_{0}$ (endowed with the norm $\left.\left\|\left(g_{k}\right)\right\|=\sup _{k}\left\|g_{k}\right\|_{v}\right)$. Since $(H v)_{0}(\Omega) \subset H v(\Omega)$ this implies Lemma 4.1. (Notice, both inclusions are inclusions as closed subspaces.)

Now we show the central

TheOREM 4.2. Let $f: \mathrm{D} \rightarrow \mathrm{C}$ be harmonic. Then $f$ is bounded if and only if $T_{f}$ is bounded. In this case there are universal constants $d_{1}, d_{2}>0$ (independent of $f$ ) such that

$$
\begin{aligned}
d_{1} M_{\infty}(f, 1) & \leq \inf \left\{\left\|T_{f}+K\right\|: K: H v(\mathrm{D}) \rightarrow H v(\mathrm{D}) \text { linear, compact }\right\} \\
& \leq\left\|T_{f}\right\| \leq d_{2} M_{\infty}(f, 1)
\end{aligned}
$$

Proof. At first let $f$ be bounded. Then $f$ has $L_{\infty}(\partial \mathrm{D})$-boundary values. According to Theorem 2.1 we obtain

$$
\left\|T_{f}\right\| \leq c \sup _{k} M_{\infty}\left(\sigma_{n_{k}}|f|, r_{m_{k}}\right)=c M_{\infty}(|f|, 1)=c M_{\infty}(f, 1) .
$$

Conversely, let $T_{f}$ be bounded. Put $f\left(r e^{i \varphi}\right)=\sum_{k \in Z} \alpha_{k} r^{|k|} e^{i k \varphi}$. Fix $n \in Z_{+}$. Then we have with $z=r e^{i \varphi}$,

$$
\begin{aligned}
z^{n} f(z)= & \sum_{k=-\infty}^{-n-1} \alpha_{k} r^{2 n} r^{|k|-n} e^{-i(|k|-n) \varphi} \\
& +\sum_{k=-n}^{-1} \alpha_{k} r^{2|k|} r^{n+k} e^{i(n+k) \varphi}+\sum_{k=0}^{\infty} \alpha_{k} r^{k+n} e^{i(k+n) \varphi}
\end{aligned}
$$

Definition 1.2 implies, with $h_{n}(z)=z^{n}$,

$$
T_{f}\left(h_{n}\right)=\sum_{k=-n}^{-1} \alpha_{k} \gamma_{k}(n) r^{n+k} e^{i(n+k) \varphi}+\sum_{k=0}^{\infty} \alpha_{k} r^{k+n} e^{i(k+n) \varphi},
$$

where, for $\left[m_{j}\right] \leq n+k<\left[m_{j+1}\right]$ and $k<0$,

$$
\gamma_{k}(n)=\frac{\left[m_{j+1}\right]-(n+k)}{\left[m_{j+1}\right]-\left[m_{j}\right]} r_{m_{j}}^{2|k|}+\frac{n+k-\left[m_{j}\right]}{\left[m_{j+1}\right]-\left[m_{j}\right]} r_{m_{j+1}}^{2|k|} .
$$


Since $\lim _{j \rightarrow \infty} r_{m_{j}}=1$ we obtain $\lim _{n \rightarrow \infty} \gamma_{k}(n)=1$ for each $k$. This implies

$$
\begin{aligned}
\left(V_{2 n, n}\right. & \left.-V_{n, 0}\right) T_{f}\left(h_{n}\right) \\
& =\sum_{k=-n}^{-1} \alpha_{k} \frac{n+k}{n} \gamma_{k}(n) r^{n+k} e^{i(n+k) \varphi}+\sum_{k=0}^{n} \alpha_{k} \frac{n-k}{n} r^{k+n} e^{i(k+n) \varphi} \\
& =z^{n}\left(\sum_{j=1}^{n} \alpha_{-j} \frac{n-j}{n} \gamma_{-j}(n) r^{-j} e^{-i j \varphi}+\sum_{k=0}^{n} \alpha_{k} \frac{n-k}{n} r^{k} e^{i k \varphi}\right) .
\end{aligned}
$$

We have $\left\|h_{n}\right\|_{v}=r_{n}^{n} v\left(r_{n}\right)$. Let $K: H v(\mathrm{D}) \rightarrow H v(\mathrm{D})$ be linear and compact. Then we obtain a universal constant $c>0$ (independent of $K, f$ and $n$ ) such that

$$
\begin{aligned}
\left\|T_{f}+K\right\| & \geq\left\|T_{f}\left(\frac{h_{n}}{\left\|h_{n}\right\|_{v}}\right)\right\|_{v}-\left\|K\left(\frac{h_{n}}{\left\|h_{n}\right\|_{v}}\right)\right\|_{v} \\
& \geq c \frac{\left\|\left(V_{2 n, n}-V_{n, 0}\right) T_{f}\left(h_{n}\right)\right\|_{v}}{\left\|h_{n}\right\|_{v}}-\left\|K\left(\frac{h_{n}}{\left\|h_{n}\right\|_{v}}\right)\right\|_{v} \\
& \geq c \frac{M_{\infty}\left(\left(V_{2 n, n}-V_{n, 0}\right) T_{f}\left(h_{n}\right), r_{n}\right)}{r_{n}^{n} v\left(r_{n}\right)}-\left\|K\left(\frac{h_{n}}{\left\|h_{n}\right\|_{v}}\right)\right\|_{v} \\
& =c \sup _{\varphi} \mid \sum_{k=1}^{n} \alpha_{-k} \frac{n-k}{n} \gamma_{-k}(n) r_{n}^{-k} e^{-i k \varphi} \\
& +\sum_{k=0}^{n} \alpha_{k} \frac{n-k}{n} r_{n}^{k} e^{i k \varphi} \mid-\left\|K\left(\frac{h_{n}}{\left\|h_{n}\right\|_{v}}\right)\right\|_{v}
\end{aligned}
$$

If we fix $m \in \mathbf{Z}_{+}$and take $n \geq m$ then we also have

$$
\begin{aligned}
\left\|T_{f}+K\right\| \geq c \sup _{\varphi} \mid \sum_{k=1}^{m} \alpha_{-k} & \frac{n-k}{n} \frac{m-k}{m} \gamma_{-k}(n) r_{n}^{-k} e^{-i k \varphi} \\
& \quad+\sum_{k=0}^{m} \alpha_{k} \frac{n-k}{n} \frac{m-k}{m} r^{k} e^{i k \varphi} \mid-\left\|K\left(\frac{h_{n}}{\left\|h_{n}\right\|_{v}}\right)\right\|_{v}
\end{aligned}
$$

Letting $n \rightarrow \infty$ Lemma 4.1 implies $\lim _{n \rightarrow \infty}\left\|K\left(h_{n} /\left\|h_{n}\right\|_{v}\right)\right\|_{v}=0$ since $K$ is compact. We arrive at

$$
\left\|T_{f}+K\right\| \geq c \sup _{\varphi}\left|\sum_{k=1}^{m} \alpha_{-k} \frac{m-k}{m} e^{-i k \varphi}+\sum_{k=0}^{m} \alpha_{k} \frac{m-k}{m} e^{i k \varphi}\right|=c M_{\infty}\left(\sigma_{m} f, 1\right)
$$


and hence

$$
c M_{\infty}(f, 1)=c \sup _{m} M_{\infty}\left(\sigma_{m} f, 1\right) \leq\left\|T_{f}+K\right\|
$$

This proves

$$
c M_{\infty}(f, 1) \leq \inf \left\{\left\|T_{f}+K\right\|: K: H v(\mathrm{D}) \rightarrow H v(\mathrm{D}) \text { linear and compact }\right\} .
$$

Theorem 2.1 yields $\left\|T_{f}\right\| \leq c M_{\infty}(f, 1)$ in view of the maximum principle.

Corollary 4.3. Let $f: \mathrm{D} \rightarrow \mathrm{C}$ be harmonic such that $T_{f}$ is compact. Then $f(z)=0$ for all $z \in \mathrm{D}$.

Recall that, according to Corollary 2.4 , for many $f$, we can replace $T_{f}$ by $T_{g}$ up to compact perturbations where $g$ is harmonic. (In the terminology of Corollary $2.4, g=f_{h}$.)

Lemma 4.4. Let $f, g: \overline{\mathrm{D}} \rightarrow \mathrm{C}$ satisfy (2.1) and assume that $f$ and $g$ are continuous on $\overline{\mathrm{D}} \backslash \rho \mathrm{D}$ for some $0<\rho<1$. Then $T_{f} T_{g}-T_{f g}$ is compact.

Proof. In view of Proposition 2.3 and Lemma 2.5 it suffices to assume that $f(z)=\alpha z^{n}$ and $g(z)=\beta z^{m}$ for some $\alpha, \beta \in \mathrm{C}$ and $m, n \in \mathrm{Z}$. If $h \in H v$ (D) is such that $h(z)=\sum_{k \geq|m|+|n|} \alpha_{k} z^{k}$ for some $\alpha_{k}$ then $T_{f} T_{g} h-T_{f g} h=0$. This means that $T_{f} T_{g}-T_{f g}$ has finite rank and hence is compact.

THEOREM 4.5. Let $f: \overline{\mathrm{D}} \rightarrow \mathrm{C}$ satisfy (2.1) and assume that $f$ is continuous on $\overline{\mathrm{D}} \backslash \rho \mathrm{D}$ for some $0<\rho<1$. Then the essential spectrum of $T_{f}$ is equal to $f(\partial \mathrm{D})$. Moreover there are constants $c, d>0$ (independent of $f$ ) such that

$$
\begin{aligned}
c M_{\infty}(f, 1) & \leq \inf \left\{\left\|T_{f}+K\right\|: K: H v(\mathrm{D}) \rightarrow H v(\mathrm{D}) \text { linear, compact }\right\} \\
& \leq d M_{\infty}(f, 1)
\end{aligned}
$$

Proof. Let

$$
\mathscr{B}=\{T: H v(\mathrm{D}) \rightarrow H v(\mathrm{D}): T \text { linear and bounded }\}
$$

and $\mathscr{K}=\{K \in \mathscr{B}: K$ compact $\}$. Then, by Lemma 4.4, the algebra $\mathscr{A}_{f}$ generated by $T_{f}+\mathscr{K}$ in $\mathscr{B} / \mathscr{K}$ is commutative. By Theorem 4.2 and Corollary 2.4 its norm is equivalent to $M_{\infty}(\cdot, 1)$. Hence $\mathscr{A}_{f}$ is a function algebra and the spectrum of $T_{f}+\mathscr{K}$ in $\mathscr{A}_{f}$ is equal to $f(\partial \mathrm{D})$.

\section{REFERENCES}

1. Lusky, W., On the isomorphism classes of weighted spaces of harmonic and holomorphic functions, Studia Math. 175 (1) (2006), 19-45. 
2. Lusky, W., and Taskinen, J., Bounded holomorphic projections for exponentially decreasing weights, to appear in J. Funct. Spaces Appl.

3. Shields, A. L., and Williams, D. L., Bounded projections, duality and multipliers in spaces of analytic functions, Trans. Amer. Math. Soc. 162 (1971), 287-302.

FAC. FOR INF. AND APPL. MATH.

UNIVERSITY OF YEREVAN

ALEK MANUKIAN 1

YEREVAN 25

ARMENIA

E-mail: anahit@ysu.am
INST. FOR MATH.

UNIVERSITY OF PADERBORN

WARBURGER STR. 100

D-33098 PADERBORN

GERMANY

E-mail: lusky@uni-paderborn.de 